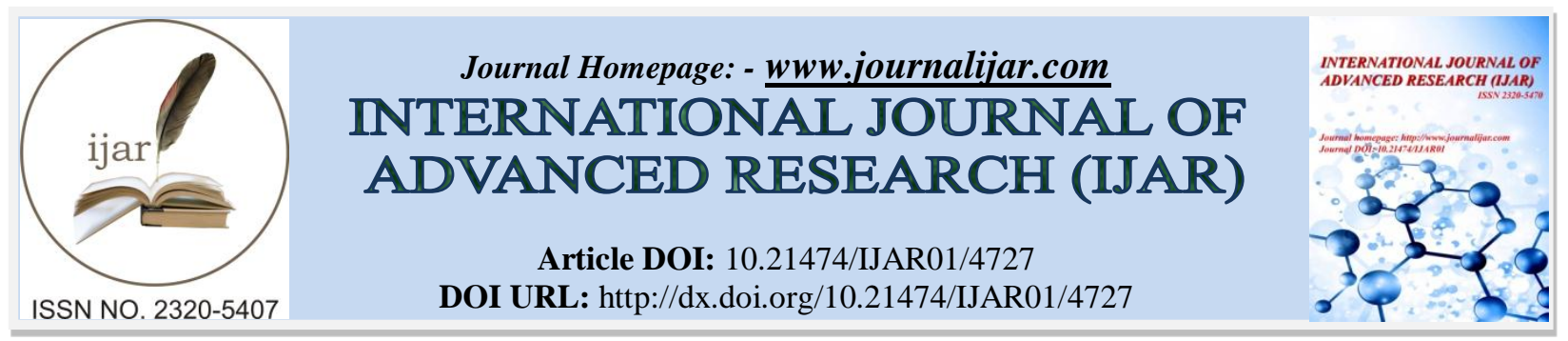

RESEARCH ARTICLE

\title{
YOUNGSTAR'S PURCHASING PATTERN/BEHAVIORS OF UNIVERSITY STUDENTS-A QUALITATIVE ANALYSIS.
}

Harshajit Singha M. Sc., PGDSMA and Ramesh Athe M. Phil., Ph. D.

Indian Statistical Institute, North East Centre, Tezpur, Assam-784028, India.

\section{Manuscript Info}

Manuscript History

Received: 4 May 2017

Final Accepted: 6 June 2017

Published: July 2017

Key words:-

University Students, Purchasing Pattern, Purchasing Behavior and Qualitative Analysis.

\begin{abstract}
A consumer's buying behavior is influenced by cultural, social, personal and psychological factors. Most of the purchases are done by youth specially students. This study carried out for the behavior of youth and which has reflected in interesting results towards the strength of rupee in the market. University life is a challenging period especially for students who have to leave their familiar surroundings and settle in a new environment. Assess the current purchasing behavior/patterns of products among youngsters of Tezpur University students at various courses. The unfamiliar environment may have an impact on their personality, attitudes and behaviour. The survey consisted of questions directly related to purchasing habits. The students were sampled by using convenience sampling method and we administered a selfreported questionnaire. A total of 366 participants PG students took part in the study. Out of 366 participants 221 (60.2\%) Male candidates, 145 (39.5\%) Female candidates were considered. We considered 15\% dropout rate for our analysis. Descriptive statistics such as frequencies and percentage were used. Pearson Chi-Square was performed to compare the different associations between variables. All reported probability values were compared to a significant level of 0.05 . We observed that there is significant association for reason for selecting brand, company, commodities and source of information and rest of them insignificant. Data entry and statistical analysis was performed with the IBM SPSS, Version 22.
\end{abstract}

Copy Right, IJAR, 2017,. All rights reserved.

\section{Introduction:-}

It is well proved that youth have very special role in any countries development. Everyday people use a variety goods and materials from the food we eat and the cloths we wear to things like cell phones, computers etc. this is consumption. Citizens of consumer countries are subject to advertising that promotes the buying of more and more things, making them feel inadequate unless they buy in. A consumer's buying behavior is influenced by cultural, social, personal and psychological factors. Most of the purchases are done by youth specially students. University life is a challenging period especially for students who have to leave their familiar surroundings and settle in a new environment. The unfamiliar environment may have an impact on their personality, attitudes and behaviour. The intention of this study is to present a statistical approach to daily essentials brands purchasing pattern of youngster's in Tezpur University (TU) students. The purpose of the study is to analyze the behaviours of the students towards 
daily essentials brands from the viewpoints of fashion apparels, mobile phones, price, quality and purchasing criteria. This study is about the purchasing behavior on Indian brands vs. foreign brands among TU students.

Various factors influencing the purchasing behavior like satisfaction, comfort, price, durability, quality, social status, country of origin etc. This study helps to throw light on the need to improve the quality of Indian brands and incorporate the emotion of "Be Indian Buy Indian" to help tide over the over dependence on multinational brands. In today's retail environment there are some who enjoy purchasing and some who do not. People have many options when it comes to where they shop; they can shop from home or venture out to the store. Purchasing has never been as fast and convenient as it is today. Technology is more advanced and internet usage is rapidly increasing. Retailers are realizing internet purchasing is a phenomenon that is not going to disappear. It is a rare retailer who has no web presence. In order to satisfy customers, managers need to examine how people shop. College students have their own purchasing behaviour as this study examines. Regardless of whether or not someone enjoys purchasing, regardless of motivation, and regardless of location or method, on some level everyone shops. Today's society is bombarded with new ways to shop.

\section{Methodology:-}

A questionnaire was developed and the survey consisted of questions directly related to purchasing habits. It was administered to post graduate students at a TU in the Assam, India. First a secondary study has done on the daily essentials purchasing behavior in India through the internet. Then we are preparing a questionnaire to collect primary data from Postgraduate degree students of TU regarding the purchasing behavior. Self-reported questionnaire was used to gather data on purchasing/behavior habits. Participation was voluntary, and informed consent was obtained from each participant. All the study participants were assured full confidentiality of the data collected. The students were sampled by using convenience sampling method and we administered a self-reported questionnaire. Objective of the Study is assessment of current purchasing behavior/patterns of products among youngsters of TU students at different physiological groups.

This primary data we are going to collect using structured questionnaire based on different factors like quality, price, social status, country of origin, brand, comfort etc. and then we will analyze using statistical tools. In this study, there were specific variables tested. Among the most interesting was a gender comparison with who liked to shop, which indicated a majority of both men and women enjoy shopping.

\section{Forming of Questionnaire:-}

The questionnaire was prepared for the study based on some factors like price, quality, country of origin, brand etc. The main focus was on the objective of the study while preparing questionnaire consists of two parts- personal data and consumption data. Open-ended questionnaire were applied in this study. Personal data includes age, sex, occupation, annual income, roll no, department, course. These questionnaires were prepared to understand more about youngster's purchasing behaviour towards Indian vs. Foreign brands.

\section{Data Collection:-}

Questionnaire method was used in this study, with self-administered and hand delivered questionnaires distributed to the students respondents. A sample of 400 Post-Graduate students has been selected by purposive sampling method. The Post-Graduate students covered in the study are from Humanities and Social Sciences, Sciences, and others (like MBA, PGDSMA etc.). The tool designed to elicit study information in order to identify the factors including socio-economic factors which are liable to affect the information seeking behaviour such as nature of the information need, the purpose it would serve or the existence of external barriers posed by the environment of PostGraduate students in the TU. As the study population was scattered and a large population was involved, the questionnaire method was adopted for collecting study data keeping in view of the objectives of the study. Objective of the Study is assessment of current purchasing behavior/patterns of products among youngsters of TU students at different physiological groups.

\section{Statistical Analysis:-}

IBM SPSS, Version 22 was used to analyze data obtained from the questionnaire, and to calculate descriptive statistics of the focus group sample. Pearson Chi-Square was performed to compare the different associations between variables. All reported probability values were compared to a significant level of 0.05 and $<0.05$ was considered as statistically significant. 


\section{Results:-}

A total of 366 participants PG students took part in the study. Out of 366 participants 221 (60.2\%) Male candidates, $145(39.5 \%)$ Female candidates were considered. We considered $15 \%$ dropout rate for our analysis. From the data obtained, the mean age of Male was 23.240 \pm 1.6710 Female was $22.766 \pm 1.1667$ years.

\section{Purchasing behavior of male and female students:-}

From table 1, Out of 221 Male participants, 107(29.2\%) participants purchased Indian brands, 94(25.7\%) participants purchased foreign brands and 20(5.5\%) participants purchased both the brands. Out of 145 Female participants, 74(20.2\%) participants purchased Indian brands, 22(6\%) participants purchased foreign brands and 49(13.4\%) participants purchased both the brands. Out of 221 Male participants, 221(57.7\%) participants purchased online, 9(2.5\%) participants did not purchased online. Female participants, 135(36.9\%) participants purchased online, $9(2.5 \%)$ participants did not purchased online. Out of 221 Male participants, $86(23.5 \%)$ participants' average monthly expenditure is $0-500,71(19.4 \%)$ participants' average monthly expenditure is $501-1000$, 39(10.7\%) participants' average monthly expenditure is 1001-1500 and 23(6.3\%) participants' average monthly expenditure is above 1501. Out of 145 Female participants, 41(11.2\%) participants' average monthly expenditure is 0-500, 71(19.4\%) participants' average monthly expenditure is 501-1000, 23(6.3\%) participants' average monthly expenditure is 1001-1500 and 7(1.9\%) participants' average monthly expenditure is above 1501 .

Out of 221 Male participants, 14(3.8\%) participants select a brand for fashion accessories based on brand loyalty, 94(25.7\%) participants select a brand for fashion accessories based on quality, 4(1.1\%) participants select a brand for fashion accessories based on availability, 23(6.3\%) participants select a brand for fashion accessories based on value for money, $12(3.3 \%)$ participants select a brand for fashion accessories based on style, 5(1.4\%) participants select a brand for fashion accessories based on other reasons and 69(18.9\%) participants select a brand for fashion accessories based on more than one reason.

\section{Brands and online purchasing:-}

From the table 2, we find that for purchasing Indian brands, 167(45.6\%) participants purchase online and 12(3.3\%) participants do not purchase online. For purchasing foreign brands, 112(30.6\%) participants purchase online and 4(1.1\%) participants do not purchase online. For purchasing both brands, 67(18.3\%) participants purchase online and $2(.5 \%)$ participants do not purchase online.

\section{Brands and average monthly expenditure:-}

From the table 3, we find that, for purchasing Indian brands, 74(20.2\%) participants' average monthly expenditure lies in 0-500, 67(18.3\%) participants' average monthly expenditure lies in 501-1000, 20(5.5\%) participants' average monthly expenditure lies in 1001-1500 and 15(4.1\%) participants' average monthly expenditure is above 1501. For purchasing foreign brands, 36(9.8\%) participants' average monthly expenditure lies in 0-500, 42(11.5\%) participants' average monthly expenditure lies in 501-1000, 26(7.1\%) participants' average monthly expenditure lies in 1001-1500 and 12(3.3\%) participants' average monthly expenditure is above 1501. For purchasing both the brands, 17(4.6\%) participants' average monthly expenditure lies in 0-500, 33(9\%) participants' average monthly expenditure lies in 501-1000, 16(4.4\%) participants' average monthly expenditure lies in 1001-1500 and 3(0.8\%) participants' average monthly expenditure is above 1501 . Since the p-value $=0.008<0.05$, so we conclude that there is an association between brands and average monthly expenditure.

\section{Purchasing Patterns:-}

As per the self-reported questionnaires, we observed that there is significant association for reason for selecting brand, company, commodities and source of information. Others like price, durability, frequency of purchase, online/offline, quality and manufactures were insignificantly associated with Indian \& Non-Indian brands.

\section{Limitation:-}

Generalizations of these findings should be approached with thoughtfulness. The sample was a convenience sample controlled in the form of an anonymous assessment to students at university. It is endorsed that additional study be conducted of a larger and more representative university student sample, to shed further light on the shopping behavior/patterns of students. 


\section{Discussion:-}

The purpose of this explorative study was to identify determinants of purchasing behaviours in TU students. Furthermore, we aimed to collect ideas and recommendations in order to facilitate the development of effective and tailored intervention programs aiming to improve purchasing behaviours in university students.

The research conducted found most college students surveyed enjoy shopping. In the study, female respondents indicated they enjoy shopping at a greater percentage than do males. The research suggests the most popular item for purchase both in-stores and online was a clothing. For their shopping experience, most respondents like to have a shopping companion, who according to the majority of respondents was a family member. Respondents also indicated they desire someone else's opinion (most of the time provided by their shopping companion) on their clothing selections. Students who do not like to shop usually shop by themselves and may seek the opinion of sales clerks relative to their purchase intentions.

Even though today's economy is not booming, respondents indicated they enjoy shopping and continue to patronize stores. The internet is indeed becoming increasingly popular, and respondents indicated they are more likely to shop in-stores as opposed to on the internet. However, respondents do consider online shopping to be more convenient, user-friendly, cheaper, quicker, and easier. It remains to be seen whether or not online shopping will overcome store shopping altogether, but due to the responses in the current study this seems unlikely especially as regards shopping for clothing. Due to shopping being a source of entertainment for many individuals, the traditional store will likely never be obsolete, but may diminish in number as internet retailers continue to increase their market presence and share.

Table1:- Purchasing behavior of male students and female students.

\begin{tabular}{|c|c|c|c|c|}
\hline Question & Sex & Male & Female & Total \\
\hline \multirow{4}{*}{ Brand } & Indian & $107(29.2 \%)$ & $74(20.2 \%)$ & $181(49.5 \%)$ \\
\hline & Foreign & $94(25.7 \%)$ & $22(6 \%)$ & $116(31.7 \%)$ \\
\hline & Both & $20(5.5 \%)$ & $49(13.4 \%)$ & $69(18.9 \%)$ \\
\hline & Total & $221(60.4 \%)$ & $145(39.6 \%)$ & $366(100 \%)$ \\
\hline \multirow[t]{3}{*}{ Online Purchasing } & Yes & $211(57.7 \%)$ & $135(36.9 \%)$ & $346(94.5 \%)$ \\
\hline & No & $9(2.5 \%)$ & $9(2.5 \%)$ & $18(4.9 \%)$ \\
\hline & Total & $221(60.4 \%)$ & $145(39.6 \%)$ & $366(100 \%)$ \\
\hline \multirow{5}{*}{$\begin{array}{l}\text { Average } \\
\text { expenditure }\end{array}$} & $0-500$ & $86(23.5 \%)$ & $41(11.2 \%)$ & $127(34.7 \%)$ \\
\hline & 501-1000 & $71(19.4 \%)$ & $71(19.4 \%)$ & $142(38.8 \%)$ \\
\hline & 1001-1500 & $39(10.7 \%)$ & $23(6.3 \%)$ & $62(16.9 \%)$ \\
\hline & Above 1501 & $23(6.3 \%)$ & $7(1.9 \%)$ & $30(8.2 \%)$ \\
\hline & Total & $221(60.4 \%)$ & $145(39.6 \%)$ & $366(100 \%)$ \\
\hline \multirow{8}{*}{$\begin{array}{l}\text { Reason of selecting a } \\
\text { brand }\end{array}$} & Brand loyalty & $14(3.8 \%)$ & $7(1.9 \%)$ & $21(5.7 \%)$ \\
\hline & Quality & $94(25.7 \%)$ & $70(19.1 \%)$ & $164(44.8 \%)$ \\
\hline & Availability & $4(1.1 \%)$ & $0(0.0 \%)$ & $4(1.1 \%)$ \\
\hline & Value for money & $23(6.3 \%)$ & $9(2.5 \%)$ & $32(8.7 \%)$ \\
\hline & Style & $12(3.3 \%)$ & $8(2.2 \%)$ & $20(5.5 \%)$ \\
\hline & Others & $5(1.4 \%)$ & $6(1.6 \%)$ & $11(3 \%)$ \\
\hline & More than one & $69(18.9 \%)$ & $44(12 \%)$ & $113(30.9 \%)$ \\
\hline & Total & $221(60.4 \%)$ & $145(39.6 \%)$ & $366(100 \%)$ \\
\hline \multirow{4}{*}{ Price } & Lowest & $22(6 \%)$ & $9(2.5 \%)$ & $31(8.5 \%)$ \\
\hline & Average & $138(37.7 \%)$ & $105(28.7 \%)$ & $243(66.4 \%)$ \\
\hline & Highest & $60(16.4 \%)$ & $31(8.5 \%)$ & $91(24.9 \%)$ \\
\hline & Total & $221(60.4 \%)$ & $145(39.6 \%)$ & $366(100 \%)$ \\
\hline \multirow{6}{*}{ Brand } & Lowest & $34(9.3 \%)$ & $20(5.5 \%)$ & $54(14.8 \%)$ \\
\hline & Average & $125(34.2 \%)$ & $95(26 \%)$ & $220(60.1 \%)$ \\
\hline & Highest & $61(16.7 \%)$ & $29(7.9 \%)$ & $90(24.6 \%)$ \\
\hline & Total & $221(60.4 \%)$ & $145(39.6 \%)$ & $366(100 \%)$ \\
\hline & Lowest & $31(8.5 \%)$ & $12(3.3 \%)$ & $43(11.7 \%)$ \\
\hline & Average & $36(9.8 \%)$ & $32(8.7 \%)$ & $68(18.6 \%)$ \\
\hline
\end{tabular}




\begin{tabular}{|c|c|c|c|c|}
\hline \multirow[t]{2}{*}{ Quality } & Highest & $153(41.8 \%)$ & $101(27.6 \%)$ & $254(69.4 \%)$ \\
\hline & Total & $221(60.4 \%)$ & $145(39.6 \%)$ & $366(100 \%)$ \\
\hline \multirow{4}{*}{ Comfort } & Lowest & $30(8.2 \%)$ & $13(3.6 \%)$ & $43(11.7 \%)$ \\
\hline & Average & $53(14.5 \%)$ & $16(4.4 \%)$ & $69(18.9 \%)$ \\
\hline & Highest & $137(37.4 \%)$ & $116(31.7 \%)$ & $253(69.1 \%)$ \\
\hline & Total & $221(60.4 \%)$ & $145(39.6 \%)$ & $366(100 \%)$ \\
\hline \multirow{4}{*}{ Style } & Lowest & $27(7.4 \%)$ & $11(3 \%)$ & $38(10.4 \%)$ \\
\hline & Average & $113(30.9 \%)$ & $64(17.5 \%)$ & $177(48.4 \%)$ \\
\hline & Highest & $80(21.9 \%)$ & $69(18.9 \%)$ & $149(40.7 \%)$ \\
\hline & Total & $221(60.4 \%)$ & $145(39.6 \%)$ & $366(100 \%)$ \\
\hline \multirow{4}{*}{$\begin{array}{l}\text { Durability and easy } \\
\text { care }\end{array}$} & Lowest & $24(6.6 \%)$ & $17(4.6 \%)$ & $41(11.2 \%)$ \\
\hline & Average & $108(29.5 \%)$ & $56(15.3 \%)$ & $164(44.8 \%)$ \\
\hline & Highest & $88(24 \%)$ & $71(19.4 \%)$ & $159(43.4 \%)$ \\
\hline & Total & $221(60.4 \%)$ & $145(39.6 \%)$ & $366(100 \%)$ \\
\hline \multirow{5}{*}{$\begin{array}{l}\text { Frequency of } \\
\text { purchase }\end{array}$} & Once in 3 months & $103(28.1 \%)$ & $39(10.7 \%)$ & $142(38.8 \%)$ \\
\hline & Once in a months & $58(15.8 \%)$ & $53(14.5 \%)$ & $111(30.3 \%)$ \\
\hline & Once in 2 months & $45(12.3 \%)$ & $43(11.7 \%)$ & $88(24 \%)$ \\
\hline & > once a month & $14(3.87 \%)$ & $10(2.7 \%)$ & $24(6.6 \%)$ \\
\hline & Total & $221(60.4 \%)$ & $145(39.6 \%)$ & $366(100 \%)$ \\
\hline \multirow{5}{*}{ Uses of internet } & Less than 1 hour & $172(47 \%)$ & $97(26.5 \%)$ & $269(73.5 \%)$ \\
\hline & 1-2 hours & $38(10.4 \%)$ & $33(9 \%)$ & 71(19.4\%) \\
\hline & 2-3 hours & $1(.3 \%)$ & $8(2.2 \%)$ & $9(2.5 \%)$ \\
\hline & $>3$ hours & $9(2.5 \%)$ & $7(1.9 \%)$ & $16(4.4 \%)$ \\
\hline & Total & $221(60.4 \%)$ & $145(39.6 \%)$ & $366(100 \%)$ \\
\hline \multirow{3}{*}{$\begin{array}{l}\text { Shopping at same } \\
\text { companies }\end{array}$} & Yes & $43(11.7 \%)$ & $24(6.6 \%)$ & $67(18.3 \%)$ \\
\hline & No & $176(48.1 \%)$ & $121(33.1 \%)$ & $297(81.1 \%)$ \\
\hline & Total & $221(60.4 \%)$ & $145(39.6 \%)$ & $366(100 \%)$ \\
\hline \multirow{7}{*}{ Bags/wallets } & Malls & $18(4.9 \%)$ & $17(4.6 \%)$ & $35(9.6 \%)$ \\
\hline & Streets & $27(7.4 \%)$ & $14(3.8 \%)$ & $41(11.9 \%)$ \\
\hline & Shops & $85(23.2 \%)$ & $73(19.9 \%)$ & $158(43.2 \%)$ \\
\hline & Online & $64(17.5 \%)$ & $25(6.8 \%)$ & $89(24.3 \%)$ \\
\hline & Showroom & $15(4.1 \%)$ & $7(1.9 \%)$ & $22(6 \%)$ \\
\hline & More than one & $12(3.3 \%)$ & $8(2.2 \%)$ & $20(5.5 \%)$ \\
\hline & Total & $221(60.4 \%)$ & $145(39.6 \%)$ & $366(100 \%)$ \\
\hline \multirow{7}{*}{ Belts } & Malls & $20(5.5 \%)$ & $10(2.7 \%)$ & $30(8.2 \%)$ \\
\hline & Streets & $39(10.7 \%)$ & $31(8.5 \%)$ & $70(9.1 \%)$ \\
\hline & Shops & $108(29.5 \%)$ & $80(21.9 \%)$ & $188(51.4 \%)$ \\
\hline & Online & $29(7.9 \%)$ & $9(2.5 \%)$ & $38(10.4 \%)$ \\
\hline & Showroom & $16(4.4 \%)$ & $9(2.5 \%)$ & $25(6.8 \%)$ \\
\hline & More than one & $6(1.6 \%)$ & $5(1.4 \%)$ & $11(3 \%)$ \\
\hline & Total & $221(60.4 \%)$ & $145(39.6 \%)$ & $366(100 \%)$ \\
\hline \multirow{7}{*}{ Fashion jewelry } & Malls & $18(4.9 \%)$ & $11(3 \%)$ & $29(7.9 \%)$ \\
\hline & Streets & $13(3.6 \%)$ & $22(6 \%)$ & $35(9.6 \%)$ \\
\hline & Shops & $70(9.1 \%)$ & $74(20.2 \%)$ & $144(39.3 \%)$ \\
\hline & Online & $36(9.8 \%)$ & $12(3.3 \%)$ & $48(13.1 \%)$ \\
\hline & Showroom & $46(12.6 \%)$ & $16(4.4 \%)$ & $62(16.9 \%)$ \\
\hline & More than one & $5(1.4 \%)$ & $9(2.5 \%)$ & $14(3.8 \%)$ \\
\hline & Total & $221(60.4 \%)$ & $145(39.6 \%)$ & $366(100 \%)$ \\
\hline \multirow{5}{*}{ Hats/caps } & Malls & $19(5.2 \%)$ & $10(2.7 \%)$ & $29(7.9 \%)$ \\
\hline & Streets & $58(15.8 \%)$ & $47(12.8 \%)$ & $105(28.7 \%)$ \\
\hline & Shops & $72(19.7 \%)$ & $62(16.9 \%)$ & $134(36.6 \%)$ \\
\hline & Online & $35(9.6 \%)$ & $14(3.8 \%)$ & $49(13.4 \%)$ \\
\hline & Showroom & $16(4.4 \%)$ & $3(.8 \%)$ & $19(5.2 \%)$ \\
\hline
\end{tabular}




\begin{tabular}{|c|c|c|c|c|}
\hline & More than one & $9(2.5 \%)$ & $5(1.4 \%)$ & $14(3.8 \%)$ \\
\hline & Total & $221(60.4 \%)$ & $145(39.6 \%)$ & $366(100 \%)$ \\
\hline \multirow{7}{*}{ Watches } & Malls & $8(2.2 \%)$ & $4(1.1 \%)$ & $12(3.3 \%)$ \\
\hline & Streets & $3(.8 \%)$ & $2(.5 \%)$ & $5(1.4 \%)$ \\
\hline & Shops & $39(10.7 \%)$ & $29(7.9 \%)$ & $68(18.6 \%)$ \\
\hline & Online & $89(24.3 \%)$ & $40(10.9 \%)$ & $129(35.2 \%)$ \\
\hline & Showroom & $64(17.5 \%)$ & $63(17.2 \%)$ & $127(34.7 \%)$ \\
\hline & More than one & $13(3.6 \%)$ & $6(1.6 \%)$ & $19(5.2 \%)$ \\
\hline & Total & $221(60.4 \%)$ & $145(39.6 \%)$ & $366(100 \%)$ \\
\hline \multirow{7}{*}{ Sunglasses } & Malls & $11(3 \%)$ & $13(3.6 \%)$ & $24(6.6 \%)$ \\
\hline & Streets & $16(4.4 \%)$ & $6(1.6 \%)$ & $22(6 \%)$ \\
\hline & Shops & $58(15.8 \%)$ & $47(12.8 \%)$ & $105(28.7 \%)$ \\
\hline & Online & $74(20.2 \%)$ & $22(6 \%)$ & $96(26.2 \%)$ \\
\hline & Showroom & $43(11.7 \%)$ & $48(13.1 \%)$ & $91(24.9 \%)$ \\
\hline & More than one & $8(2.2 \%)$ & $6(1.6 \%)$ & $14(3.8 \%)$ \\
\hline & Total & $221(60.4 \%)$ & $145(39.6 \%)$ & $366(100 \%)$ \\
\hline \multirow{7}{*}{ Mobile phone } & Malls & $3(.8 \%)$ & $1(.3 \%)$ & $4(1.1 \%)$ \\
\hline & Streets & $2(.5 \%)$ & $2(.5 \%)$ & $4(1.1 \%)$ \\
\hline & Shops & $38(10.4 \%)$ & $30(8.2 \%)$ & $68(18.6 \%)$ \\
\hline & Online & $93(25.4 \%)$ & $40(10.9 \%)$ & $133(36.3 \%)$ \\
\hline & Showroom & $69(18.9 \%)$ & $66(18 \%)$ & $135(36.9 \%)$ \\
\hline & More than one & $15(4.1 \%)$ & $5(1.4 \%)$ & $20(5.5 \%)$ \\
\hline & Total & $221(60.4 \%)$ & $145(39.6 \%)$ & $366(100 \%)$ \\
\hline \multirow{3}{*}{ Uses of smartphone } & Yes & $202(55.2 \%)$ & $142(38.8 \%)$ & $344(94 \%)$ \\
\hline & No & $19(5.2 \%)$ & $3(.8 \%)$ & $22(6 \%)$ \\
\hline & Total & $221(60.4 \%)$ & $145(39.6 \%)$ & $366(100 \%)$ \\
\hline \multirow{4}{*}{ Design } & Most important & $60(16.4 \%)$ & $57(15.6 \%)$ & $117(32 \%)$ \\
\hline & Average & $127(34.7 \%)$ & $79(21.6 \%)$ & $206(56.3 \%)$ \\
\hline & Least important & $34(9.3 \%)$ & $9(2.5 \%)$ & $43(11.7 \%)$ \\
\hline & Total & $221(60.4 \%)$ & $145(39.6 \%)$ & $366(100 \%)$ \\
\hline \multirow{4}{*}{ Sound quality } & Most important & $107(29.2 \%)$ & $94(25.7 \%)$ & $201(54.9 \%)$ \\
\hline & Average & $96(26.2 \%)$ & $45(12.3 \%)$ & $141(38.5 \%)$ \\
\hline & Least important & $18(4.9 \%)$ & $6(1.6 \%)$ & $24(6.6 \%)$ \\
\hline & Total & $221(60.4 \%)$ & $145(39.6 \%)$ & $366(100 \%)$ \\
\hline \multirow{4}{*}{ Brand } & Most important & $127(34.7 \%)$ & $99(27 \%)$ & $226(61.7 \%)$ \\
\hline & Average & $79(21.6 \%)$ & $42(11.5 \%)$ & $121(33.1 \%)$ \\
\hline & Least important & $15(4.1 \%)$ & $4(1.1 \%)$ & $19(5.2 \%)$ \\
\hline & Total & $221(60.4 \%)$ & $145(39.6 \%)$ & $366(100 \%)$ \\
\hline \multirow{4}{*}{ Video quality } & Most important & $107(29.2 \%)$ & $88(24 \%)$ & $195(53.3 \%)$ \\
\hline & Average & $96(26.2 \%)$ & $48(13.1 \%)$ & $144(39.3 \%)$ \\
\hline & Least important & $18(4.9 \%)$ & $9(2.5 \%)$ & $27(7.4 \%)$ \\
\hline & Total & $221(60.4 \%)$ & $145(39.6 \%)$ & $366(100 \%)$ \\
\hline \multirow{4}{*}{ Price } & Most important & $107(29.2 \%)$ & $77(21 \%)$ & $184(50.3 \%)$ \\
\hline & Average & $105(28.7 \%)$ & $62(16.9 \%)$ & $167(45.6 \%)$ \\
\hline & Least important & $9(2.5 \%)$ & $6(1.6 \%)$ & $15(4.1 \%)$ \\
\hline & Total & $221(60.4 \%)$ & $145(39.6 \%)$ & $366(100 \%)$ \\
\hline \multirow{4}{*}{ Processor } & Most important & $168(45.9 \%)$ & $107(29.2 \%)$ & $275(75.1 \%)$ \\
\hline & Average & $46(12.6 \%)$ & $31(8.5 \%)$ & $77(21 \%)$ \\
\hline & Least important & $7(1.9 \%)$ & $7(1.9 \%)$ & $14(3.8 \%)$ \\
\hline & Total & $221(60.4 \%)$ & $145(39.6 \%)$ & $366(100 \%)$ \\
\hline \multirow[b]{3}{*}{ Screen size } & Most important & $51(13.9 \%)$ & $425(11.5 \%)$ & $93(25.4 \%)$ \\
\hline & Average & $146(39.9 \%)$ & $90(24.6 \%)$ & $236(64.5 \% \$)$ \\
\hline & Least important & $24(6.6 \%)$ & $13(3.6 \%)$ & $37(10.1 \%)$ \\
\hline
\end{tabular}




\begin{tabular}{|c|c|c|c|c|}
\hline & Total & $221(60.4 \%)$ & $145(39.6 \%)$ & $366(100 \%)$ \\
\hline \multirow{4}{*}{$\begin{array}{l}\text { Battery } \\
\text { life }\end{array}$} & Most important & $179(48.9 \%)$ & $123(33.6 \%)$ & $302(82.5 \%)$ \\
\hline & Average & $36(9.8 \%)$ & $18(4.9 \%)$ & $54(14.8 \%)$ \\
\hline & Least important & $6(1.6 \%)$ & $4(1.1 \%)$ & $10(2.7 \%)$ \\
\hline & Total & $221(60.4 \%)$ & $145(39.6 \%)$ & $366(100 \%)$ \\
\hline \multirow{4}{*}{ Camera } & Most important & $134(36.6 \%)$ & $110(30.1 \%)$ & $244(66.7 \%)$ \\
\hline & Average & $70(19.1 \%)$ & $29(7.9 \%)$ & $99(27 \%)$ \\
\hline & Least important & $17(4.6 \%)$ & $6(1.6 \%)$ & $23(6.3 \%)$ \\
\hline & Total & $221(60.4 \%)$ & $145(39.6 \%)$ & $366(100 \%)$ \\
\hline \multirow{4}{*}{ RAM } & Most important & $182(49.7 \%)$ & $116(31.7 \%)$ & $298(81.4 \%)$ \\
\hline & Average & $34(9.3 \%)$ & $23(6.3 \%)$ & $57(15.6 \%)$ \\
\hline & Least important & $5(1.4 \%)$ & $6(1.6 \%)$ & $11(3 \%)$ \\
\hline & Total & $221(60.4 \%)$ & $145(39.6 \%)$ & $366(100 \%)$ \\
\hline \multirow{3}{*}{$\begin{array}{l}\text { Interest in leather } \\
\text { fashion }\end{array}$} & Yes & $104(28.4 \%)$ & $77(21 \%)$ & $181(49.5 \%)$ \\
\hline & No & $114(31.1 \%)$ & $68(18.6 \%)$ & $182(49.7 \%)$ \\
\hline & Total & $221(60.4 \%)$ & $145(39.6 \%)$ & $366(100 \%)$ \\
\hline \multirow{3}{*}{ Scholarship } & Yes & $60(16.4 \%)$ & $42(11.5 \%)$ & $102(27.9 \%)$ \\
\hline & No & $161(44 \%)$ & $102(27.9 \%)$ & $263(71.9 \%)$ \\
\hline & Total & $221(60.4 \%)$ & $145(39.6 \%)$ & $366(100 \%)$ \\
\hline \multirow{6}{*}{$\begin{array}{l}\text { Sources } \\
\text { information }\end{array}$} & Internet & $131(35.8 \%)$ & $97(26.5 \%)$ & $228(62.3 \%)$ \\
\hline & Friends & $14(3.8 \%)$ & $17(4.6 \%)$ & $31(8.5 \%)$ \\
\hline & Advertisements & $5(1.4 \%)$ & $2(.5 \%)$ & $7(1.9 \%)$ \\
\hline & Others & $3(.8 \%)$ & $3(.8 \%)$ & $6(1.6 \%)$ \\
\hline & More than one & $66(18 \%)$ & $26(7.1 \%)$ & $92(25.1 \%)$ \\
\hline & Total & $221(60.4 \%)$ & $145(39.6 \%)$ & $366(100 \%)$ \\
\hline \multirow{6}{*}{$\begin{array}{l}\text { Occasion } \\
\text { purchasing }\end{array}$} & Festivals & $52(14.2 \%)$ & $45(12.3 \%)$ & $97(26.5 \%)$ \\
\hline & Offers & $60(16.4 \%)$ & $45(12.3 \%)$ & $105(28.7 \%)$ \\
\hline & Gifts & $5(1.4 \%)$ & $2(.5 \%)$ & $7(1.9 \%)$ \\
\hline & Others & $57(15.6 \%)$ & $29(7.9 \%)$ & $86(23.5 \%)$ \\
\hline & More than one & $47(12.8 \%)$ & $24(6.6 \%)$ & $71(19.4 \%)$ \\
\hline & Total & $221(60.4 \%)$ & $145(39.6 \%)$ & $366(100 \%)$ \\
\hline
\end{tabular}

Table2:- Brands vs. online purchasing.

\begin{tabular}{|l|l|l|l|l|l|l|}
\hline \multirow{3}{*}{$\begin{array}{l}\text { Online } \\
\text { purchasing }\end{array}$} & Brands & Indian & \multicolumn{1}{|c|}{ Foreign } & \multicolumn{1}{|c|}{ Both } & \multicolumn{1}{|c|}{ Total } & \multirow{2}{*}{-value } \\
\cline { 2 - 6 } & Yes & $167(45.6 \%)$ & $112(30.6 \%)$ & $67(18.3 \%)$ & $346(94.5 \%)$ & \\
\cline { 2 - 6 } & No & $12(3.3 \%)$ & $4(1.1 \%)$ & $2(.5 \%)$ & $18(4.9 \%)$ & \\
\cline { 2 - 6 } & Total & $181(49.5 \%)$ & $116(31.7 \%)$ & $69(18.9 \%)$ & $366(100 \%)$ & \\
\hline
\end{tabular}

Table 3:- Brands vs. average monthly expenditure.

\begin{tabular}{|l|l|l|l|l|l|l|}
\hline \multirow{4}{*}{$\begin{array}{l}\text { Average } \\
\text { monthly } \\
\text { expenditure }\end{array}$} & Brands & Indian & \multicolumn{1}{|c|}{ Foreign } & \multicolumn{1}{|c|}{ Both } & \multicolumn{1}{|c|}{ Total } & p-value \\
\cline { 2 - 6 } & $\mathbf{0 - 5 0 0}$ & $74(20.2 \%)$ & $36(9.8 \%)$ & $17(4.6 \%)$ & $127(34.7 \%)$ & \\
\cline { 2 - 6 } & $\mathbf{5 0 1 - 1 0 0 0}$ & $67(18.3 \%)$ & $42(11.5 \%)$ & $33(9 \%)$ & $142(38.8 \%)$ & \\
\cline { 2 - 6 } & $\mathbf{1 0 0 1 - 1 5 0 0}$ & $20(5.5 \%)$ & $26(7.1 \%)$ & $16(4.4 \%)$ & $62(16.9 \%)$ & \\
\cline { 2 - 6 } & Above 1501 & $15(4.1 \%)$ & $12(3.3 \%$ & $3(.8 \%)$ & $30(8.2 \%)$ & \\
\cline { 2 - 6 } & Total & $181(49.5 \%)$ & $116(31.7 \%)$ & $69(18.9 \%)$ & $366(100 \%)$ & \\
\hline
\end{tabular}

*indicates statistically significant at $5 \%$ level of significance.

Conflicts of interest: There are no conflicts of interest.

Ethics: Ethical approval was not required for the present study.

Authors' contributions: Author 1 received the M.Sc. degree from Tezpur University and PG Diploma in Statistical Methods (PDGSMA) from Indian Statistical Institute, North East Centre, Tezpur. H.S. contributed in the data collection, analysis and manuscript preparation.

Author 2 received the M.Phil., and Ph.D. (NIN, ICMR) in Statistics awarded from Osmania University, Hyderabad. R.A. developed the study protocol, supervised the study and guided manuscript preparation. 


\section{Acknowledgements:-}

The authors thank Prof. S. Bandyopadhyay (Director, Indian Statistical Institute) for her encouragement \& carrying out the study, and also Dr M Vishnu Vardhana Rao (NIN, ICMR, Hyderabad), Prof. N. Sarkar, and Dr CH. Holendro Singh (Indian Statistical Institute, North East Centre) for their insightful inputs.

\section{References:-}

1. Abheek S., Nimisha J., Kanika S. (2017). The new Indian: The many facets of changing consumer. BCG Centre for consumer insight.

2. Amitabh M. et al. (2012). The tiger roars: capturing India's explosive growth in consumer spending. BCG Centre for consumer insight.

3. Archana Kumar, et al. (2007). Indian consumer's purchase behaviour towards US versus local brands. International Journal of Retail and Distribution Management. 37(6), pp.510-526.

4. Babbie, E. (1992). The Practice of Social Research, Sixth Edition, Wordsworth, Belmont, California.

5. Berman B and Evans J (2002), Retail Management: A Strategic Approach, Pearson education, Asia, Singapore.

6. Batra, R., et al. (2000). Affects of brand domestic and non domestic origin on consumer attitudes in developing countries", Journal of Consumer Psychology, 9(2), pp.83-85.

7. Bhuvaneswari V. and Jayasree K. (2015). A review of literature on impulse buying behaviour of consumers in bricks \& mortar and click only stores. IJMSSR, Vol. 2, No.3, pp. 84-90.

8. Biswas R. (2006). India"s changing consumer", Chain Store Age, 82(5), p.A2.

9. Clark, R.A., and Goldsmith, R.E. (2005). Spreading their Sales. Business India Intelligence.12(6), pp.1-2.

10. Emilija A. et al. (2010). University students shopping patterns internet vs. brick and mortar. The Journal of Applied Business Research, Vol. 26, No. 1, pp. 31-36.

11. Gopal A. and Srinivasan R. (2006). The Indian Consumer", Harvard Business Review, 84 (10), pp.22-23.

12. Guha S. (2013). The changing perception and buying behaviour of women consumer in Urban India. IOSRJBM, Pp. 34-39.

13. Gupta A. (2004). Factors Affecting the Trial of Private Labels in Departmental Store: Evidence from India. The ICFAI Journal of Brand Management, Vol. 1, No. 3, pp. 6-21.

14. Naresh N.P. et al. (2015). Online shopping patterns amidst students with respect to food products. IJMSSR, Vol. 4, No.9, pp. 26-33.

15. ncdrc.nic.in (National Consumer Disputes Redressal Commission.)

16. Ott B. (2005. Is There a Digital Divide in Online Shopping? Retrieved March 7, 2009, from Gallup: http://www.gallup.com/poll/20527.

17. Pallavi K. (2012). Changing purchasing behaviour of Indian customers. APJEM, Vol. 1, No. 8, pp. 35-41.

18. Prasad G.H.S. (2014). Factors Influencing Buying Behavior of a Selected Apparel Retailer's Customers. Annual Research Journal of Symbiosis Centre for Management Studies, Pune Vol. 1, pp. 41-55.

19. Rai M.K. and Gopal R. (2016). Study of consumer buying behavior on branded ethnic apparel. IOSR-JBM, Pp. 43-49.

20. Shailesh K.K. (2013). The importance of apparel product attributes for teenaged buyers. NMIMS, Management Review.

21. Suchita G. (2014). Consumer attitude and purchase behavior towards foreign brands. IJRM, Vol. 2, Issue 1, pp. 74-87. 